\title{
Key Techniques Research in MOOCs
}

\author{
Weijun DONG, Guohua GENG
}

\author{
School of information and technology, Northwest University, Xi'an China 710127 \\ wjdong@nwu.edu.cn,ghgeng@nwu.edu.cn
}

\section{Key Words: MOOC; CORBA; CSCW;}

Abstract: Massive Online Open Courses(MOOC) which based on the computer technology and network technology integrates many quality education resources. The construction of MOOC curriculum resources platform is more important than the construction of cource resources. Analysising the characters of MOOC, design and proposal a new MOOC Curriculum Resources system which based on many years experiences of MOOC construction. and use the technique of Event server of CORBA. New system provides a CSCW environment which provides a framework supporting cooperation. It also can provide many interactive modes, multiple learning-feedback. It is very useful for students to achieve multiple goals and multiple developments, and can effectively improve the efficiency of Learner autonomy.

\section{Introduction}

Massive Online Open Course (MOOC) is an innovation form of Open Educational Resources (OER). In the last two years, because of its unique characteristics and the quality of resources, MOOC has attracted more and more students to participate in. All teaching activities and management activities occurred in the network environment, the learners' number, study time, region and age will not be limited in the MOOC study environment ${ }^{[1,2]}$. We can find that the core of MOOC is integrate the most high-quality educational resources of the world, achieve global sharing, provide strong support for better occupation career and realize lifelong study ${ }^{[3,4]}$. Although MOOC system has made much progress, there are some problems in use, such as: (1) the lack of effective management of personal learning process; (2) the management of learning process and program links is relatively rough; (3) learning interaction model is not fully. When the designers of MOOC design and develop the system, they have assumed that if learners spend much time and energy, they will understand all foundmental knowledge and finish the study. Which leads to those problems haven't been fundamentally resolved. Therefore, an effective MOOC curriculum resources platform not only need to complete the course teaching, but also need to provide a learning management system which can make the students more reasonably arrange their learning, realize efficient, sustainable self-study and self-development.

On the other hand, computer supported cooperative work (CSCW) technique which based on computer technology and communication technology can realize computers work together to complete a complex task ${ }^{[5,6]}$. In some ways, MOOC is a remote collaborative teaching activity relies on computer network environment, in which the teacher, student and educational institutions and other groups are linked toghter by autonomous learning acticities. So, it has a strong theoretical and practical significance that make full use of CSCW technology to build a MOOC system which suit for the characteristic of Chinese students. This paper discusses how to use CSCW technique to build a MOOC curriculum resources platform which can meet the diversified interactive, independent and individualized learning model in a perfect and dynamic way.

\section{Design of MOOC}

When we design and build a MOOC system, we must consider the autonomous learning, consider the collaborative learning and management of learning task, which can be well resolved by CSCW technolog. 


\section{Design of MOOC's functions}

The MOOC platform provides powerful resource construction and independent learning environment for teachers and students. There are each course contains 4 independent function modules which includes teaching organization and management, communication tools, management function and management statistical. Functions of MOOC resources platform is shown in figure 1.

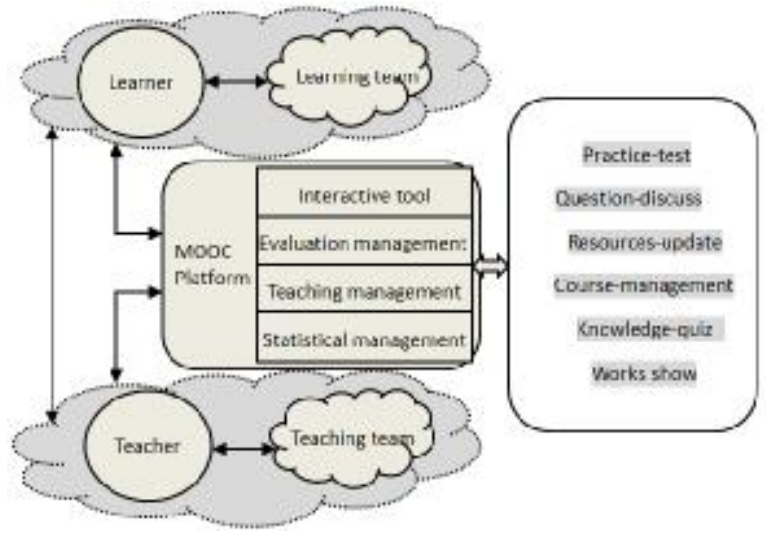

Fig.1 The function and Interactive mode of MOOC platform

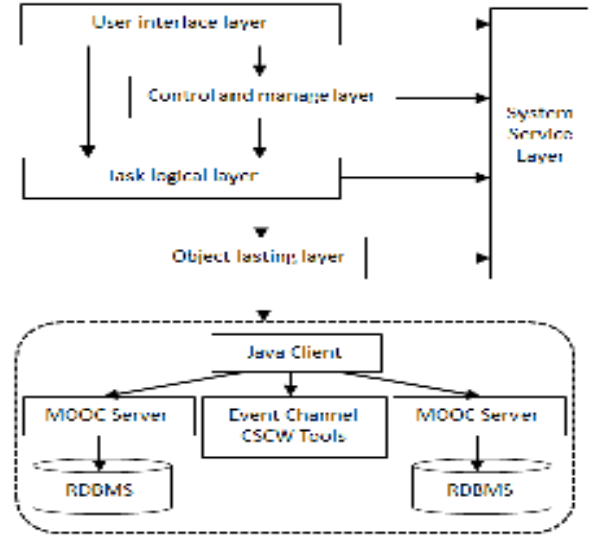

Fig.2 System architecture of MOOC

The interaction mechanism contains 5 important interaction forms: the interaction between students and learning resources, the interaction between teachers and learning resources, the interaction between teachers and students, the interaction between the learning team and student, the interaction between teacher and teaching team. The 5 interactive models can improve the effective control of the learning process, the main characteristics show as: the interaction between teachers and students, students and students are no longer single mode; teaching mode has been changed from single information dissemination to two-way interactive communication; students' study has been changed from the passive acceptance to active learning; it can carry out remote exchanges and cooperation in various forms between students, and can promote the cultivation of team spirit; through curriculum resource interactive platform whcih provides seamless integration and effective knowledge, teachers can realize one to one, many to many communication and collaboration .

Logical model of MOOC

(1) System architecture

System's architecture has an important influence on system's security and reusability. The architecture of the MOOC curriculum resources platform which use distributed multilayer structure, it is shown in fig. 2. User interface layer is composed of some interaction class, which can send messages to the task logic class, control class and system class.Control and manage layer finish task logic control through the collaboration with task logic layer. The system service layer is responsible for communicating with operating system, and requests operating system to complete the task.

Object lasting layer of the isolation between system object model and system database storage model, which realizes the access to database application package. And provides a complete interface which can provide object level operations for the task logic layer.

(2) Event channel

In the MOOC resource platform, it often appears non-coupling and asynchronous communication demand . At the same time, we want the object to send messages to relevant parties, rather than relevant parties continuously detect the object to determine whether there is any new messages can get; we also hopes that the sent should be multicast. The best way to solve this problem is to use asynchronous and non-coupling multicast communications. in an asynchronous non-coupling multicast communication environment, the event service mechanism based on the "producer consumer" communication model can provide object separation between communications, which allow multiple producer object sendind data asynchronously to multiple consumers object by event 
channel, also can make an object convey the important change of state to other objects in which the event interest.

The event channel model is shown in fig. 3.

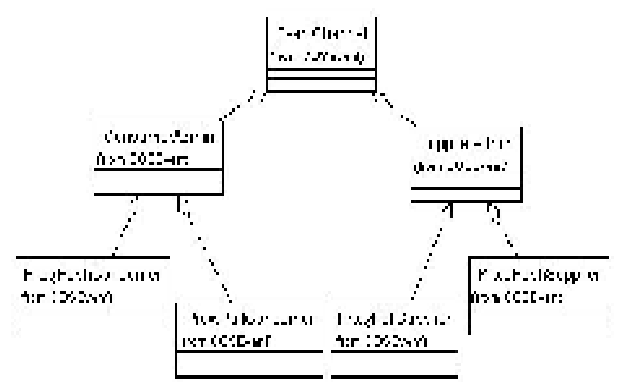

Fig.3 The model of event

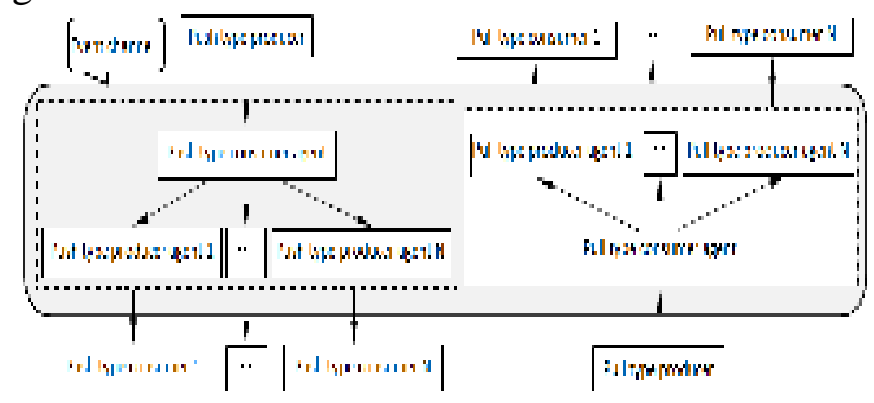

Fig.4 Event channel communication model

The event channel is an object which can receive events from multiple event producers, and delivery each event that it receives to its connected event consumers. The event channel plays an intermediary agent role between event producers and consumers, and provids an agent of interface to each kind of producers or consumers. This agent is not only inherited by proxy operation interface, but also provides some additional operations which can be used to establish and remove proxy client connections.For each client which support producer interface, will create a object which support proxy consumer interface in the event channel, and the client will be connected with the object. Similarly, for each client which support consumer interface, will create a object which support proxy produce interface in the event channel, and the client will be connected with the object.In this way, multiple producers can be connected wi th the same channel; multiple consumer also can be connected with the same channel.

(3) Communication model

The event channel shields the relationship between producers and consumers, it can receive events with push model and provide events with pull model, or receive events with pull model and provide events with push model. This means that a pull type producers can provide data to a push type consumers; a push type consumers can also provide data to a pull type producer. Event channel communication model is shown in fig. 4.

In the push model, the application which produces an event will use the event as an operation parameter, and the delivery of event is realized by calling the push operation of consumer event. The application of produce event supports the PushSuplier interface. The application of consumer event supports PushConsumer interface. In the pull model, consumer objects control data flow by producers data pulling back. The difference with the push model lies that the consumer need call the pull operation of producers, and the event will be returned as a result, at this time, event producer support PullSuplier interface, and event consumers support PullConsumer interface.

\section{Discussion}

Distributed object technology has become the core technology and standard component framework of application system in distributed and heterogeneous environment, which has a major part to play in the enterprise integration, integration of distributed system management and software component technology. There are 3 basic technical standards: CORBA, COM/DCOM and RMI. CORBA which is mainly applied to heterogeneous, large-scale distributed system is outstanding for strict ruler, independent of producer and advanced distributed computing characteristics in the 3 kinds of standard. So, when analysis and design MOOC curriculum resources platform, select CORBA as the underlying platform in distributed object computing standards.

In the implementation of the system, the formation of the framework based on the reusing of code, and provide the service interface. Frame object is responsible for interaction with the event channel, and provids event delivery service. For he object which use the framework, interacting with event channel is completely transparent transmission. 
In the push model, the application does not need to constantly detect event channel whether there are new events, it is more efficient than the pull model, so select push model as the system event processing model in two communication models of event service. Designing a f push type event delivery framework, we realize MOOC curriculum resources platform based on CSCW technology. The event service produces a loose-coupling "producer - consumer" communication mode between objects are transparent, this communication mode allows transparent data exchange at an evironment that the communication both sides do not know the status of each other. The event channel is the core of event communication, which can transparently broadcast the data that is produced by producers to consumers. Because the event communication mode is dynamic, the group members can join and leave the group dynamically and freely.

Distributed MOOC curriculum resources platform needs the support of database, the fundamental task is resolve the problem the effective model matching between object model and relation model. For this, design the object lasting layer to solve the mapping problem of object-relationship. Object lasting layer is located between the task logical layer and data storage systems, which can reduce the dependence of business model on data storage system, increase the robustness, flexibility and flexibility of the system.

\section{Conclusions}

With the delepment of MOOC, the traditional educational theory and teaching methods of university need be changed. So, the construction of MOOC curriculum resources platform has more realistic significance than the construction of cource resources. At the same time, the values of distributed object computing is becoming more and more important with the increasing geometricly of internet resources. The main driving force of distributed system is how to efficiencily combine a large number of personal computer and people work together with sharing information, this combination should be the precondition for facility and easy management, and it can not be limited by physical distribution and of geographical,personnel data or machines.

MOOC curriculum resources platform which supports push type events model based on CSCW technology select CORBA as the distributed computing platform. Curriculum resources platform can optimize the network education resources, can make teach and learn more efficiency, scientificity and practicality, students also can study, experimentand quiz on this platform according to their own independent selection, so as to improve theirlearning interest, and achieve good learning results.

\section{Acknowledgements}

This work was supported by research foundation of Northwest University, China

\section{References}

[1]J. Han.From PID to Active Disturbance Rejection Control[J]. Industrial Electronics, IEEE Transactions on, 2009,56(3):900 -906.

[2]J. Ligus, J. Ligusova, J. Sarnovsky, I. Zolotova, and T. Gazda.E ducation as cybernetic system[J]. EAEEIE Annual Conference,2011 Proceedings of the 22nd, 2011, 1-5.

[3]P. Bistak and P. Folvarcik. Remote laboratory java server based on jacob project[J]. International Journal of Online Engineering, 2011, 7(1):33-36.

[4]J. Ligusova, J. Ligus, and I. Zolotova. Cybernetic education centre[J].EAEEIE Annual Conference (EAEEIE), 2013 Proceedings of the 24th,2013, 133-138.

[5]Teruel Miguel,etc. Comparing goal-oriented approaches to model requirements for CSCW [J]. Communications in Computer and Information Science,2013, 27(5):169-184.

[6]Haixiao Sheng,Hongfu Zuo,Juan Xu. Study on Agent Collaborative Work Model of Aircraft Spares Support Based on CSCW Environment[J].Information Technology Journal,2013,12(11):2213 $-2218$. 